\title{
Dicerto, S. (2018). Multimodal pragmatics and translation: A new model for source text analysis. London: Palgrave Macmillan. 178 pp.
}

This book by Sara Dicerto is the latest publication in the Palgrave Studies in Translating and Interpreting series that focus on the role that translation and interpreting play in their various forms at all levels of communication nowadays, both locally and globally. Such interface studies are associated closely with neighboring disciplines such as pragmatics and multimodal communication, the main content of this book, which consists of six chapters. In the following, I will summarize and evaluate the individual chapters before providing an overall assessment.

In Chapter 1, Dicerto explains that the landscape of translation research has taken on new shapes in terms of translation theories and source text (ST) forms. As for the latter, STs are no longer confined to linguistic terms alone, but take on new forms (multimodal texts including linguistic forms, pictures, sounds etc.). After a brief introduction to existing translation theories, she shows the relationships between visual forms and linguistic forms as well as the necessity of incorporating both forms in conveying meaning. Subsequently, Dicerto outlines the purpose of this book, i.e., to explain how multimodal STs convey meaning in a multimodal context and to build a model for the pragmatic translation of multimodal STs before ascertaining the applicability of the model. In this introductory chapter, Dicerto succeeds in arousing the reader's interest by pointing out the intrinsically multimodal essence of communication, the under-researched translation of semiotic resources other than linguistic forms as well as the limited focus of the existing interface studies of pragmatics and translation.

Chapter 2 focuses mainly on (social) semiotics and the existing multimodal theoretical framework, which serves as one of the bases for the model presented in the book. Semiotics deals mainly with how different modes (as well as their disparities and similarities) are organized to make meaning. The multimodal theoretical framework mainly touches upon the interaction models for the various semiotic modes, such as proposed in Multimodal Discourse: The Modes and Media of Contemporary Communication (Kress \& Van Leeuwen, 2001) and Multimodal Transcription and Text Analysis (Baldry \& Thibault, 2005). After commenting on relevant theoretical bases, Dicerto points out that there is no universally accepted theoretical framework to organize different modes nor are there consistent "multimodal principles" to coordinate different modes in the translation of multimodal STs. She therefore considers it necessary that new theoretical insights (here pragmatic perspectives) should be incorporated to assist the translation of multimodal STs. This chapter, with detailed analysis of the roles that modes play in meaning commutation, discusses the lack of common ground in modes organization so that "communication glue" should be found elsewhere (i.e., pragmatics). By doing so, Dicerto shows again in a convincing manner the importance of her new model for translation and readers are triggered to move on to the third chapter to discover the role that pragmatics may play in the model.

Chapter 3 begins with a discussion about whether pragmatic theories can be applied to the interpretation of multimodal meaning. Dicerto's literature review reveals that pragmatic theories can, to some extent, be helpful to interpret written and oral language in traditional translation research. However, there exist spatial, temporal and cognitive disparities between writers (speakers) and readers (listeners), which may result in inadequacy of the meaning being conveyed when resorting to linguistic forms alone. Therefore, other modes are needed to clarify the implied meaning (i.e., meaning in context in pragmatics). Meanwhile, Dicerto demonstrates that relevance theory (RT) (Sperber \& Wilson,1995) and cooperative principle (CP) (Grice,1989) have been extended to the interpretation of both linguistic texts and utterances, but CP, unlike RT, has not yet been used to interpret other semiotic resources than language. Subsequently, she focuses her attention on the relationships between RT and multimodal texts. Further, Dicerto lists several cases to illustrate the adaptability of pragmatic theories (here, RT) to the 
interpretation of multimodal meaning, such as the meaning extraction in advertisement discourse. The discussion of these cases yields new insights into the application scope of the traditional pragmatic theories (here, RT), since RT research has overwhelmingly been confined to language to the best of my knowledge, although both Sperber and Wilson (1995) and Forceville (1996) have pointed out that RT could be applied to non-verbal communication. Up to now, Dicerto has paved the way theoretically, through ample literature review in Chapters 2 and 3, for building the multimodal pragmatic framework for translation, which is the key task in the next chapter, also the main part of the book.

In Chapter 4, Dicerto tries to construct a multimodal pragmatic framework for translation. The framework consists of three dimensions: (1) the analysis of pragmatic meanings conveyed by multiple modes, (2) the interaction of different modes, and (3) the interpretation of each mode. For the first dimension, with the help of RT, the author points out that conveying meaning, whether explicit or implicit, is a process by which the translator (or interpreter) extracts meaning out of multiple modes based on their optimal relevance. The underlying reason is that explicit and implicit meaning interpretation in linguistic texts depends on the semantic meaning of the texts themselves, as well as on grammatical rules. However, in multimodal texts, interpreting meaning relies mainly on the optimal relevance of the modes because the grammatical links that connect different linguistic forms in linguistic texts are simply not there to connect the different modes. In this dimension, Dicerto again reminds the reader of the necessity of pragmatic theory (i.e., RT) application to multimodal translation for RT may function as "communication glue" to incorporate all modes in multimodal ST when conveying meaning. In fact, Dicerto's "communication glue" is "visual grammar" (Kress \& Van Leeuwen, 1996) to integrate different modes when communicating meaning. In other words, RT works as a kind of rule to connect modes in multimodal texts. For the second dimension, Dicerto focuses on the semantic relationships between verbal and visual modes as well as their interactions. The relationships among these modes fall into three categories: equivalence, complementarity and independence. Dicerto illustrates what these categories mean one by one with cases that are in verbal and/or visual modes or their combination to explain the probable relationships among different modes in multimodal contexts. Translators (or interpreters) are supposed to realize how these modes interact before they start to translate or interpret. This point is worth our attention in general, since we live in a multimodal era, and especially important for sight translators or interpreters for their working environments are rich in pictures, slides, sounds, gestures, posters, postures, and video clips etc. For the third dimension, i.e., the interpretation of each mode, Dicerto states that signs can convey different meanings, express the same meaning from different perspectives or communicate a meaning in a more or less detailed way. This means that a translator (or an interpreter) is supposed to figure out the meanings of the different modes first in their multimodal translation. However, the lack of grammatical systems for visual and aural modes forces translators to look for other rules (here, optimal relevance) to coordinate, compromise and finally combine meanings that each mode may communicate. In this process, translators may have much room for interpreting visual modes and their own translation style may be easily reflected in the TT.

Dicerto's three-dimension model reveals a process of moving from a micro perspective to a macro perspective for multimodal translation. This model is hierarchical: it begins with the representation of mono-modal information (linguistic terms, visual or aural modes), then moves on to the representation of multimodal meaning (interaction of different modes and logical semantic relationships) and ends with meaning interpretation (explicit meaning and implicit meaning). The interpretation of meaning is not onedimensional and its phases are not clear-cut but a process in which different modes interact and overlap and translators (interpreters) try to figure out the STs' meaning of optimal relevance.

After theoretically establishing her model covering the multidisciplinary fields of both multimodality and pragmatics in Chapter 4, Dicerto shows how it can be applied in 
Chapter 5. She does so on three kinds of STs, using Reiss's (1989) classification: informative, expressive and operative texts. After reading her detailed delineation and explanation of the features, styles and modal organization of each kind of text, we get two insights into multimodal pragmatic translation. First, each translation activity falls into three dimensions of the model: (1) semantic representation of a single mode, (2) semantic representation of multiple modes and (3) meaning inferences. The process in which translators decide which dimension the translation of a multimodal text belongs to is how translators make TTs equivalent to STs, not only linguistically but also multi-modally. Secondly, although a translation activity can be classified as belonging to a given dimension in the model, this does not mean that this translation activity belongs to this given dimension only. It rather means that it overwhelmingly belongs to this dimension, yet other dimensions may also affect that translation activity. For example, the difficulty of rendering a multimodal ST (e.g., a picture with a specific and rich cultural meaning) into a reader-friendly TT may change the methods to reproduce STs, thereby affecting the accessibility of the pragmatic intention of ST writers. Unfortunately, Dicerto fails to touch upon this point and does not provide readers in her model with methods to avoid subjectivity as a translator or interpreter when rendering multimodal STs into TTs.

Chapter 6 further discusses the usefulness of the multimodal pragmatic model of translation. Dicerto argues that the model promotes a better understanding of STs, helps to find better solutions to the problems encountered in the translation process and helps to better reproduce STs. The model can indeed be applied as an analysis tool, not only to the translation of the texts Dicerto discusses, such as audio-visual texts and localization and case studies, but also to individual case studies, language-pair-specific issues and specific ST genres. It can also be used to analyze the special styles of some ST writers, detecting their tendency for specific lexical items and the development of their aesthetic outlook. However, compared with the paradigm of corpus-based studies of a translator's style, this model is weaker due to its subjectivity and partiality while corpus-based research will convince readers with quantitative and objective data. Although Dicerto's model can be used for training new interpreters (translators), as she herself mentions, by informing them about both the multiple modes and the interaction of ST components, it is complex and lacks practicality and operability when teachers apply the model to their teaching practice. Last but not the least, this model can be applied to a variety of fields such as literary translation, arts translation and advertisement translation, but unfortunately Dicerto does not offer readers relevant literature or examples.

When handling translation problems, readers (and by extension users) will unavoidably consider the relationship among ST components and other contextual meanings that may, more or less, relate to ST content, organization and format, factors that belong to different modes in multimodal pragmatic translation practice. In our view, the model that Dicerto has advanced in this book has yielded the following new insights into ST analysis. First, pragmatic theories (here RT) can be applied to non-verbal semiotic resources other than linguistic forms alone in translation. Second, ST components take on a variety of forms (modes) with three relationships among them (equivalence, complementarity and independence), which requires different translation strategies to get the interpretive equivalence of a TT to its ST in a multimodal context. Third, this model, in a theoretical sense, will raise translators' or interpreters' awareness to allocate textual resources and enable them to account for the development of the multimodal texts in space and time in their practice. Although the model cannot help teachers practically to guide students due to its complexity, it may be theoretically beneficial to help them reflect on their translation versions from both multimodal and pragmatic perspectives when communicating multimodal messages. Fourth, despite that Dicerto has developed the model mainly for translation, it can also be a valuable tool for the analysis of other aspects, such as language for special purposes, due to its descriptive power as well as the pervasiveness of multimodality. 
Unfortunately, the model also has its deficiencies: 1) Dicerto focuses overwhelmingly on static texts and fails to take into account dynamic texts when applying the model, which may weaken the validity or applicability of the model; 2) the model fails to elaborate on the relationships between the ST and the receivers, which limits its descriptive power; 3) this model was established using English STs and Italian TTs, two hypotaxis languages, and it therefore remains to be seen whether it can be generalized and applied to translation of and into oriental and parataxis languages (e.g., Chinese).

To sum up, the framework for multimodal pragmatic translation in this book has been established from a multidisciplinary perspective and is theoretically comprehensive. It is insightful for researchers but less successful in practice for teachers and students. It still needs further modification for practical analysis of STs (e.g., detailed guidelines for extracting each mode in STs). All in all, although the book does not offer its users and readers a panacea for all translation problems, but it is a research and analytical tool that can help them to carry out a systematic analysis of STs and anticipate potential problems in achieving equivalence both multi-modally and pragmatically between TTs and STs. Therefore, this book is truly valuable for trainers and researchers mainly for its multidisciplinary perspective, critical evaluation, comprehensive overview, meticulous case studies, well-organized format.

\section{References}

Baldry, A., \& Thibault, P. J. (2005). Multimodal transcription and text analysis. London: Equinox. Forceville, C. (1996). Pictorial metaphor in advertising. London: Routledge.

Grice, H. P. (1989). Studies in the way of words. Cambridge: MA: Harvard University Press.

Kress, G., \& van Leeuwen, T. (1996). Reading images: The grammar of visual design. London: Routledge.

Kress, G., \& van Leeuwen, T. (2001). Multimodal discourse: The modes and media of contemporary communication. London: Arnold.

Reiss, K. (1989). Text types, translation types and translation assessment. In A. Chesterman (Trans. \& Ed.), Readings in translation theory (pp. 105-115). Helsinki: Oy Finn Lectura Ab.

Sperber, D., \& Wilson, D. (1995). Relevance: Communication and cognition (2nd ed.) Oxford: Blackwell.

\section{Funding}

This work was supported by Applied Linguistics Research Project: Multidimensional and Multidisciplinary Perspectives [Grant No.: 20180101]; The China National Foundation for Social Sciences (Chinese Academic Works Translation Program) [Grant No.: 16WZS011] and The China National Foundation for Social Sciences [General Program] [Grant No.: 17BYY009 and 16XYY010].

\section{Zhanhao Jiang}

Research Center for Linguistics and Applied Linguistics/School of English Studies, Xi'an International Studies University, Xi'an, Shaanxi Province, P.R. China; jiangzhanhao@xisu.edu.cn

\section{Yuan Tao}

School of Foreign Languages, Shaanxi Normal University, Xi'an, Shaanxi Province, P.R. China taoyuanhuahua@126.com 